\title{
Shielding of Electronic Systems against Transient Electromagnetic Interferences
}

\author{
H. Herlemann ${ }^{1}$, S. Korte ${ }^{1}$, M. Camp ${ }^{1}$, H. Garbe ${ }^{1}$, M. Koch ${ }^{1}$, and F. Sabath ${ }^{2}$ \\ ${ }^{1}$ University of Hannover, Institute of Electrical Engineering and Measurement Science, Appelstr. 9a, \\ 30167 Hannover, Germany \\ ${ }^{2}$ Scientific Institute for Protection Technologies, NBC-Protection, Postfach 1142, 29623 Munster, Germany
}

\begin{abstract}
In order to protect electronic systems against the effects of transient electromagnetic interferences, shields made of electrically conductive material can be used. The subject of this paper is an electrically conductive textile. When applying the shield, a reliable measure is needed in order to determine the effectiveness of the shield to protect against electromagnetic pulses. For this purpose, a time domain measurement technique is presented using double exponential pulses. With these pulses, the susceptibility of an operating electronic device with and without the shield is determined. As a criterion of quality of a shield, the breakdown failure rate found in both cases is compared.
\end{abstract}

\section{Introduction}

Over the last years, electronic equipment has become more and more susceptible to the adverse effects of electromagnetic fields. As the geometrical dimensions of electric circuits of devices correlate with the wavelength of e.g. modern communication radio frequencies, electromagnetic waves couple more easily to the systems. With the rise in complexity, the path of coupling becomes difficult to predict. Sensitive electronic equipment is also threatened by the influence of fast transient electromagnetic fields as a result of pulsed signals. Depending on the pulse shape in time domain, the bandwidth can be very brought. Effects of these interferences can reach from interruptions of the data flow to physical destructions of semiconductors. The destruction effects of semiconductors caused by the impact of different kinds of high amplitude electromagnetic pulses have been investigated recently (Camp et al., 2002).

In Fig. 1, microscopic analysis of destructions due to these pulses are reported. At lower field strengths only electronic components like diodes or transistors on the chip, mostly as a result of flashover effects, are damaged (Fig. 1a). If the amplitude of the electromagnetic pulse increases by about $50 \%$, additional on-chip-wire destruction (this means melting of pcb tracks without flashover effects) and multiple component destruction occurred (Fig. 1b). Further increase of

Correspondence to: H. Herlemann

(herleman@geml.uni-hannover.de) the amplitude leads to additional bondwire destructions and multiple component- and on-chip wire destruction. The destructions are caused by the impact of an electromagnetic pulse (EMP) generated in a large guided wave EMP simulator. Details of the measurement setup can be found in Camp et al. (2002).

The question of protection against radiated electromagnetic fields is a question of shielding. In recent years, a variety of electrically conductive textiles has been developed for different applications in EMC (Koch, 2001). The question of how to determine the protective properties of a shield is a difficult one to be addressed, even in frequency domain. Even though it was shown in Koch (2001) that an electrical shielding effectiveness (SE) of 30-50 dB can be achieved across the frequency range from $0.1-10 \mathrm{GHz}$, in practice the protection effect of an enclosure is considerably less. This is because in most cases, only the electric- or magnetic SE of the empty shield is measured. Since the shield usually forms a cavity resonator with internal resonances, the SE breaks down at the cavity's characteristic resonance frequencies. In addition, it becomes a function of position. Due to these resonance phenomena tests as close as possible to the actual configuration are preferred over measurements of the shield alone. For these measurements, carried out in the frequency domain, a dummy load is used.

In order to assure the undisturbed operation of sensitive electronic equipment in an environment endangered by transient electromagnetic fields, i.e. electrostatic discharges (ESD), high power microwave (HPM) or ultra wideband (UWB) pulses, the knowledge of the SE in frequency domain may be not sufficient.

In this contribution, a measurement technique in the time domain is presented, using pulses of double exponential characteristic.

\section{Protective propertes of closed shields}

When equipment is inserted into a shield its resonance behaviour is changed. In addition, the coupling path of transient electromagnetic fields into the electronic becomes difficult to predict. To obtain definitive results the best way is to measure the susceptibility of the equipment with and 


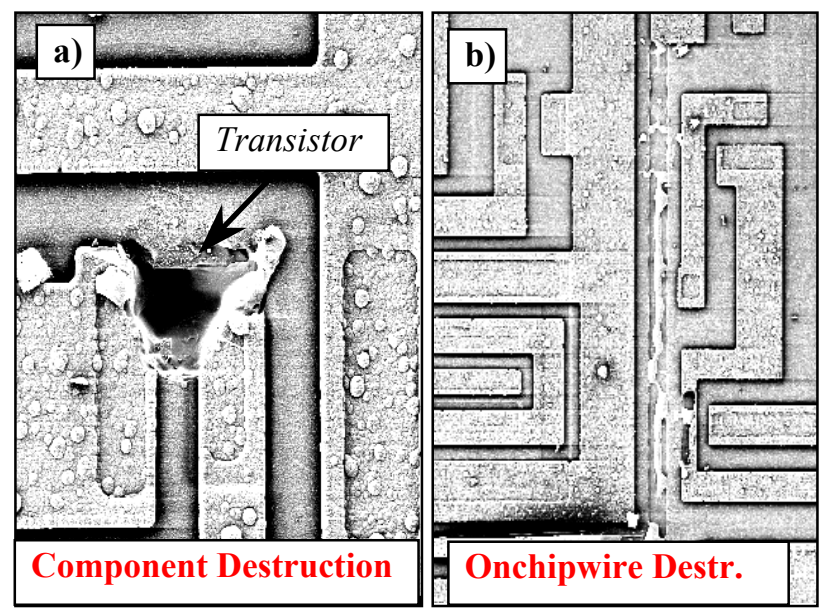

Fig. 1. Destruction Effects on Chip Level.

Table 1. Failure Rates.

\begin{tabular}{|c|c|}
\hline Breakdown & Destruction \\
\hline$\uparrow_{\text {Pulse }}^{\substack{\text { Self-, External- } \\
\text { Power-Reset }}}$ & $\uparrow_{\text {Pulse }}$ \\
\hline $\begin{array}{l}\text { Breakdown Failure } \\
\underline{\text { Rate }}\end{array}$ & $\begin{array}{l}\text { Destruction Failure } \\
\underline{\text { Rate }}\end{array}$ \\
\hline BFR $=\frac{\text { No. of Breakdowns }}{\text { No. of Pulses }}$ & DFR $=\frac{\text { No. of Destructions }}{\text { No. of Pulses }}$ \\
\hline
\end{tabular}

without the shield while being in operation. From the different field strength where certain malfunctions appear the protective properties of the shield can be obtained.

\section{Definitions}

To describe the different failure effects of electronic devices when being exposed to electromagnetic pulses, two quantities can be defined (Table 1) (Camp, 2001). The Breakdown Failure Rate (BFR) is defined as the number of breakdowns of a system, divided by the number of pulses applied to it. A breakdown means no physical damage is done to the system. After a reset (self-, external- or power reset) the system is going back into function.

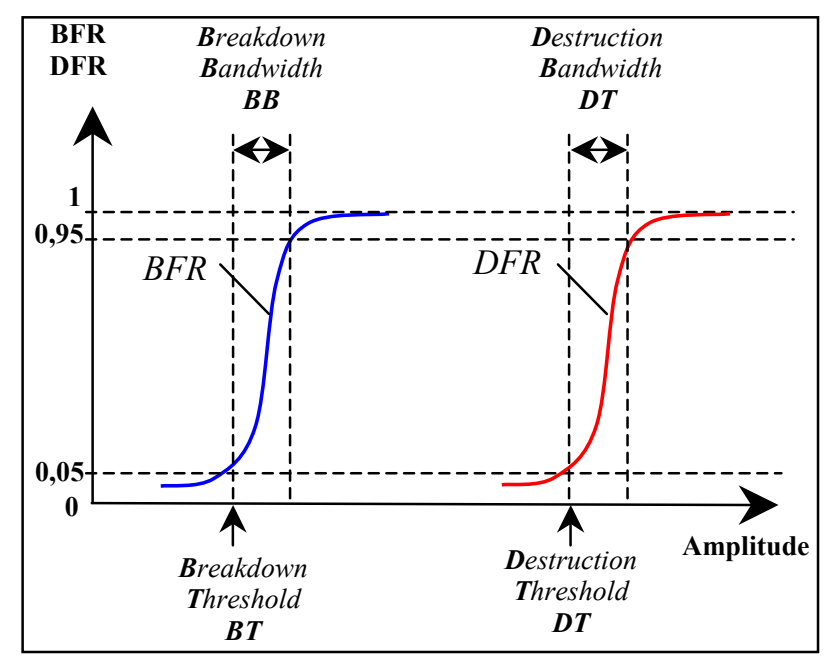

Fig. 2. BFR, DFR: Principle Behavior and Definitions.

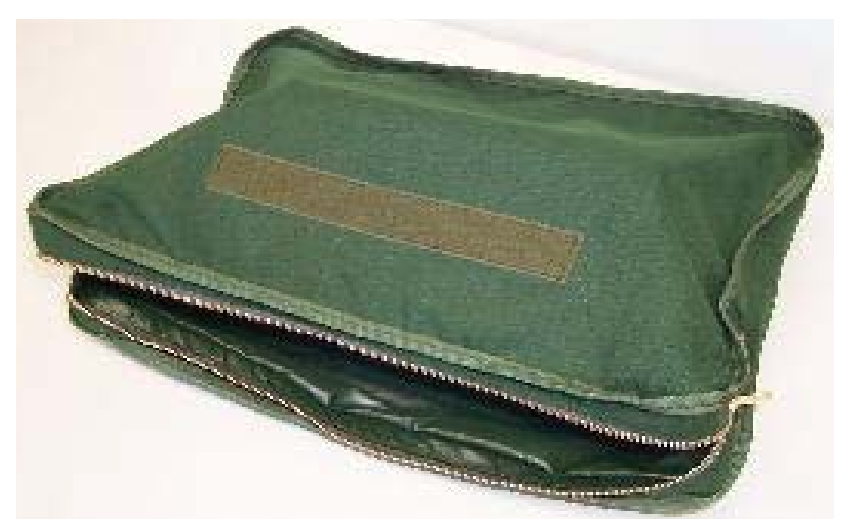

Fig. 3. Electrically Conductive Textile Pouch.

The Destruction Failure Rate (DFR) of the device under test is defined as the number of destructions divided by the number of pulses applied to the system. Destruction is defined as a physical damage of the system so that the system will not recover without a hardware repair.

The BFR and DFR behaves in principle as shown in Fig. 2. As important parameters for the description of the susceptibility of a system four quantities can be defined: The Breakdown Threshold (BT) specifies the value of the electrical field strength, at which the BFR reaches $5 \%$ of the maximum value. The Breakdown Bandwidth (BB) is defined as the span of the electrical field strength, in which the BFR changes from $5 \%$ to $95 \%$ of the maximum. Equivalent definitions are made for the destruction failure rate DFR (compare Fig. 2).

\section{Measurements}

Measurements were carried out using ultra wide band pulses to examine the influence of a protective pouch (shown in Fig. 3) on the BFR of various Microprocessorboards and 


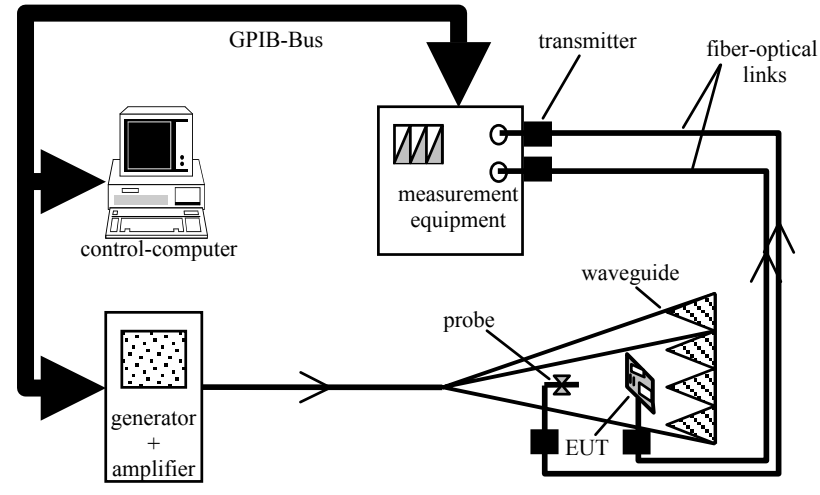

Fig. 4. Measurement Setup.

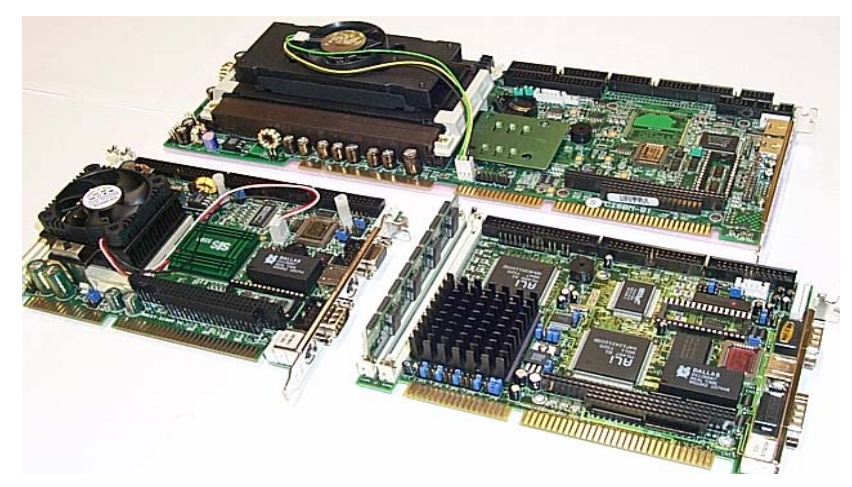

Fig. 5. Microprocessor boards.

Microcontrollers. The pulses have a double exponential form, a risetime of about $100 \mathrm{ps}$ and a full-width-at-half-max of about $2.5 \mathrm{~ns}$.

\subsection{Testsetup microprocessor boards}

The susceptibility measurements were done by a computer which controls both measurement equipment and generator/amplifier as shown in Fig. 4: The data lines of the equipment under test (EUT) and of the different field probes are fiber optical links to avoid coupling effects. The communication between the controlling computer, generator/amplifier and measurement equipment is done with the General Purpose Interface Bus (GPIB).

Different microprocessor boards as shown in Fig. 5 were tested. During the test a program was running on the board which provides a signal at a selected pin of the parallel port. This signal was monitored to ensure that the main functions of the board (processor, RAM, etc.) are still in function. After a breakdown of the board, an external reset has been carried out with a fiber optical link, to get the board back to function.

Three different microcontrollers with a different number of I/O-ports have been investigated.

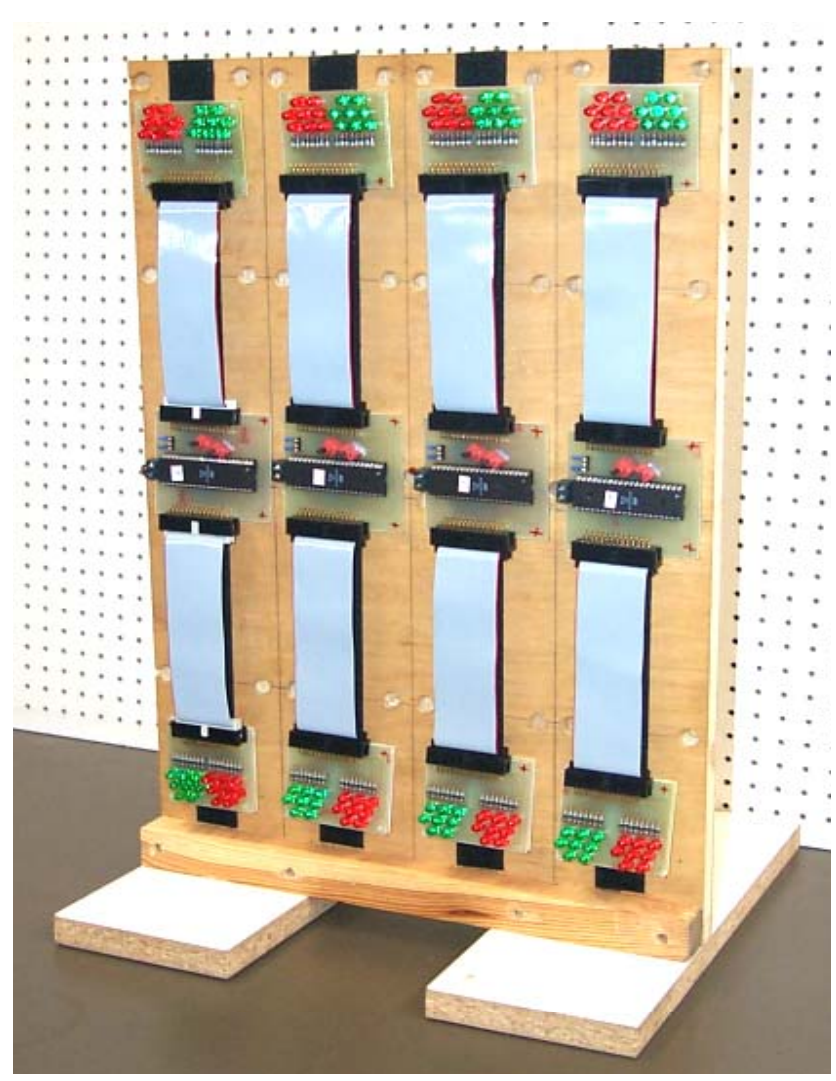

Fig. 6. Microcontroller Testsetup.

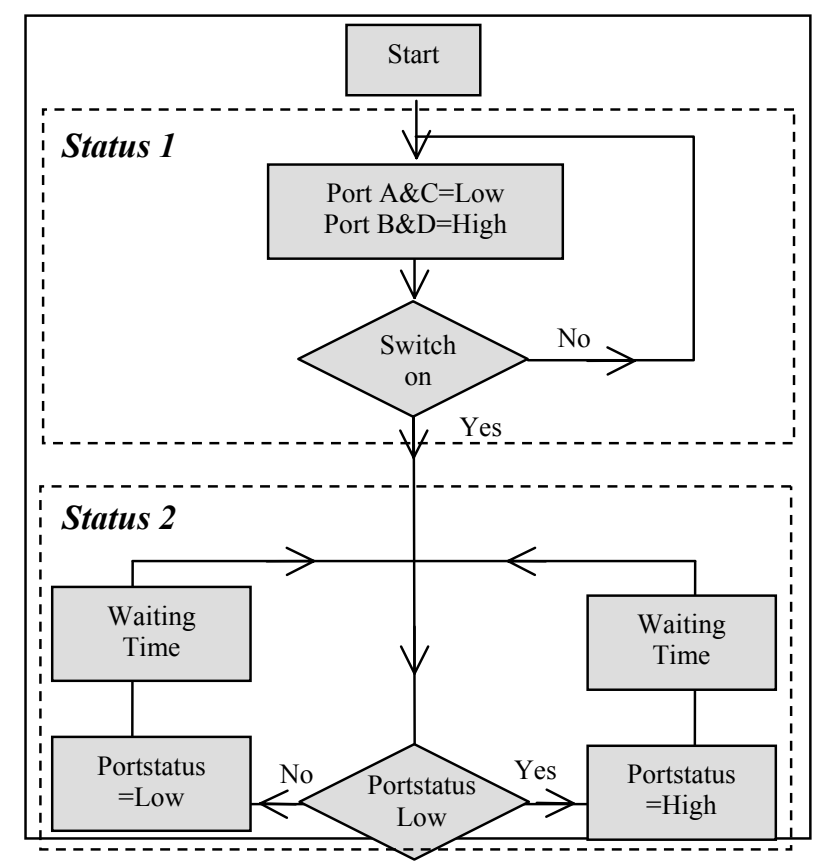

Fig. 7. Microcontroller Test Program. 


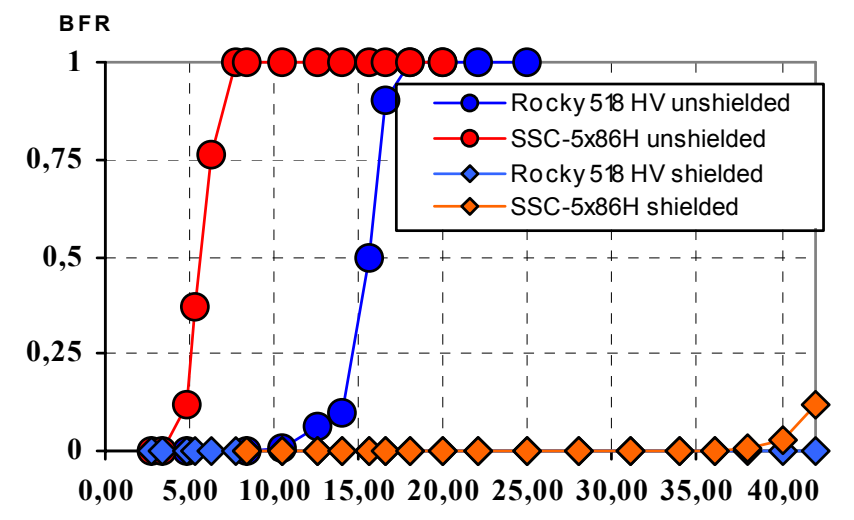

$\mathrm{E}$ in $\mathrm{kV} / \mathrm{m}$

Fig. 8. BFR of Microprocessor boards.

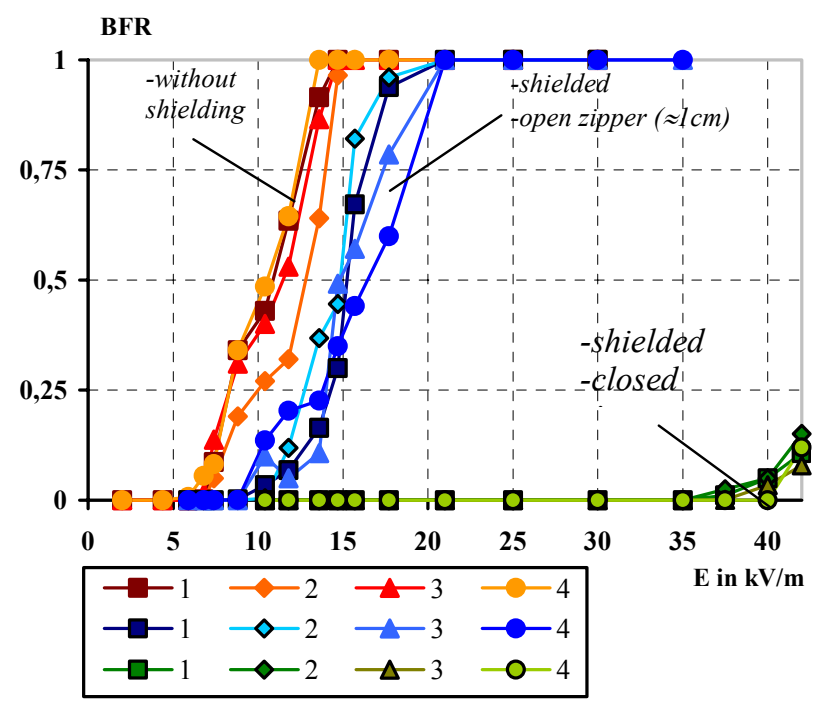

Fig. 9. BFR of Microcontroller AT90S2333.

The features of the microcontrollers are: RISC Architecture; High-speed CMOS Process Technology; $32 \times 8$ General Purpose Working Registers; Flash on Board; EEPROM on Board.

Four microcontrollers of the same type have been tested simultaneously to observe any difference. The microcontroller circuits were placed vertically on a wooden support (Fig. 6) and packed into the pouch which has been placed inside the waveguide. The different states of the I/O-ports are monitored via different coloured led's.

During the test a program was running on the microcontrollers which can get into two different states (Fig. 7): In status 1 two ports are high and two ports are low to observe this state. After a switch the program moves to the second state in which the microcontrollers were exposed to the pulses. The intention is to observe a self reset of the system by changing from status 2 back to status 1 . Without the implementation of two states a self reset cannot be observed due to the fast reset action.

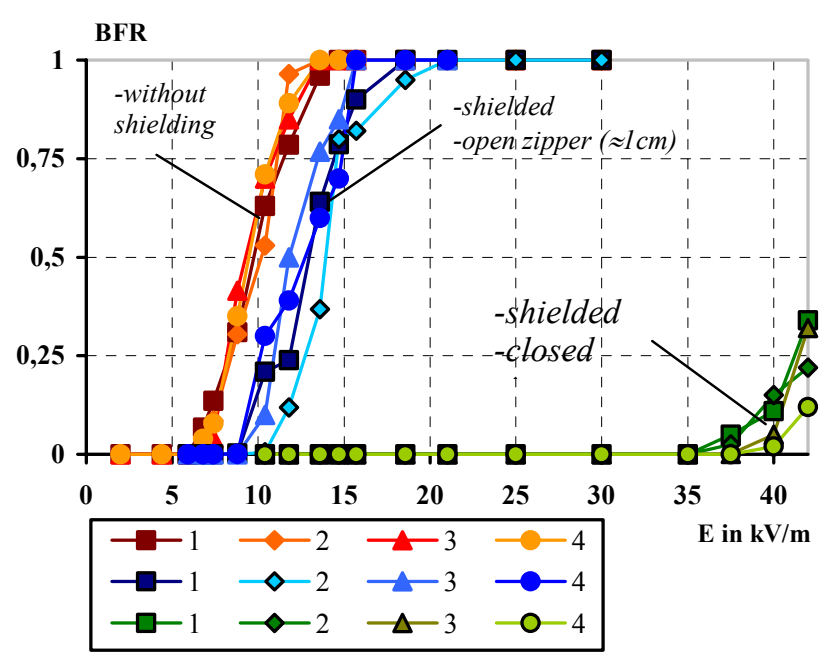

Fig. 10. BFR of Microcontroller AT90S8515.

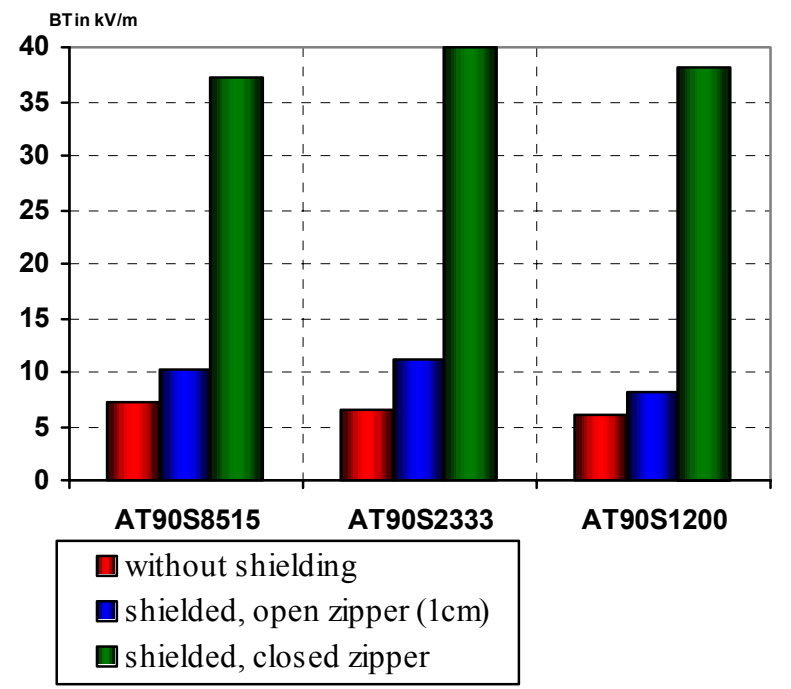

Fig. 11. BT of Microcontrollers.

\subsection{Measurement results}

The investigation of two different microprocessor boards showed a significantly higher immunity level after the boards were inserted into the pouch (Fig. 8).

In case of the unshielded SSC-5x86H board first failures occurred at $5 \mathrm{KV} / \mathrm{m}$. After inserted into the pouch this value was shifted to the eight times higher level of $40 \mathrm{kV} / \mathrm{m}$. In case of the shielded Rocky 518 HV no failures could be detected within the limits of possible field strength.

For the microcontroller measurements the procedure was slightly extended. In addition to the shielded and unshielded measurements, measurements with an unproperly closed pouch were performed: The zipper was left approx. $1 \mathrm{~cm}$ open. As mentioned before three different types or controller were tested. Four controllers of the same type were tested simultaneously. 
The results are displayed in Figs. 9 and 10 for two of the microcontrollers. Again it can be seen that the pouch with properly closed zipper shifts the curve of the breakdown failure rate to an approximately eight times higher level.

If the zipper is only partially closed, even if the remaining gap is only $1 \mathrm{~cm}$, the shielding effect decreases dramatically as can be seen in all cases. There is nearly no improvement compared to the completely unshielded case. All investigated microcontrollers displayed a similar behaviour.

The Breakdown Threshold (BT) according to Fig. 2 is shown in Fig. 11 for all three types of microcontrollers. From the graphic it can be verified that the BT is between six and eight times higher compared to the unshielded case. The improvement of the BT by factor six to eight can be identified as the protective property of the pouch.

\section{Summary}

The protective properties of a conductive textile pouch for the storage of electronic equipment were investigated. For this purpose, electronic devices were exposed to ultra wideband pulses inside a guided wave EMP simulator, while being in operation. The susceptibility of the devices with and without shield were determined. Despite the fact that different electronic devices were used to measure the shielding property of the pouch, the protection effect of the examined shield is for all examined cases approx. the same: An increase in resistance against 6 to 8 times higher field strengths. As a consequence, the shift of the breakdown threshold from the unshielded to the shielded case can be used as a criterion of quantity of a shield. Some of the included material is part of the study "Susceptibility of Electronics to EMP and UWB, Phase II", commissioned by the Armed Forces Scientific Institute for Protection Technologies - ABC-Protection (Munster, Germany).

\section{References}

Braun, C.:Aufbau eines breitbandigen Wellenleiters für NEMP Modell Simulationen, INT Bericht Okt. 1984.

Camp, M.: Empfindlichkeit von Elektronik gegen EMP und UWBPhase II, WIS Bericht, Okt. 01, AN:005.H-0 A064, AG:UT 243C, 2001.

Camp, M., Garbe, H., and Nitsch, D.: UWB and EMP Susceptibility of Modern Electronics, IEEE EMC, Montreal, August 13-17, 2001, ISBN: 0-7803-6569-0, 1015-1020, 2001.

Camp, M., Garbe, H., and Nitsch, D.: Influence of the Technology on the Destruction Effects of Semiconductors by Impact of EMP and UWB Pulses, IEEE Intl. Symp. on EMC, Minneapolis, Aug. 19-23, 2002.

IEEE Std 299-1997: IEEE Standard Method for Measuring the Effectiveness of Electromagnetic Shielding Enclosures, 1997.

Koch, M.: Applications of Electrically Conductive Textiles, 14th International Zurich Symposium on Electromagnetic Compatibility, 2001.

Nitsch, D., Schlüter, J., and Kitschke, H. J.: Generierung und Vorteile von Ultrawideband-Impulsen, EMV99, Mannheim, Germany, 1999.

Sabath, F., Nitsch, D., and Koch, M.: Messung der Reflexionsund Transmissionseigenschaften textiler Lagen im Zeit- und Frequenzbereich, EMV 2002, Tagungsband, VDE-Verlag, 2002. 